\title{
Démystification et invention du quotidien : les objets des romans de Jean Echenoz
}

\section{Florence Bouchy}

\section{Q OpenEdition}

1 Journals

Édition électronique

URL : http://journals.openedition.org/recherchestravaux/428

DOI : 10.4000/recherchestravaux.428

ISSN : 1969-6434

Éditeur

UGA Éditions/Université Grenoble Alpes

\section{Édition imprimée}

Date de publication : 20 décembre 2010

Pagination : 77-89

ISBN : 978-2-84310-187-8

ISSN : 0151-1874

Référence électronique

Florence Bouchy, « Démystification et invention du quotidien : les objets des romans de Jean Echenoz », Recherches \& Travaux [En ligne], 77 | 2010, mis en ligne le 20 août 2012, consulté le 08 septembre 2020. URL : http://journals.openedition.org/recherchestravaux/428 ; DOI : https://doi.org/ 10.4000/recherchestravaux.428 


\section{Démystification et invention du quotidien : les objets des romans de Jean Echenoz}

L'omniprésence des objets quotidiens dans les romans de Jean Echenoz a été, dès la parution de ses premiers livres ${ }^{\mathrm{I}}$, fréquemment soulignée par la critique. Jean Lebrun écrit d'ailleurs, dans la monographie parue en 1992, que " [t]els l'écume visible des habitudes et des modes de vie de notre temps, ces objets flottent ainsi à la surface de chaque livre. Pour se frayer sa voie, le récit donne sans cesse l'impression de devoir zigzaguer entre eux, rappelant le parcours de l'individu dans la société urbaine de consommation ${ }^{2} »$. Une telle saturation du texte par les marqueurs de la quotidienneté contemporaine pourrait rendre tentante la mise en évidence, dans ces romans, d'un nouveau "système des objets ${ }^{3}$ ", de portée critique, et propre à notre époque. Encore faudrait-il que la notion de "système» soit pertinente pour rendre compte $\mathrm{du}$ statut particulier qu'occupent ces objets dans l'ensemble textuel et des effets de sens majeurs qu'ils suscitent. Comme le notait déjà, d'ailleurs, Gérard Genette, la question «n'est pas tant de savoir s'il y a ou non un système de relations dans tel ou tel objet de recherches, puisqu'il y en a évidemment partout, mais de déterminer l'importance relative de ce système par rapport aux autres éléments de compréhension $[\ldots]^{4} »$. La lecture sémiologique systématique qui prendrait appui sur le relevé et l'analyse des objets d'un roman parait en fait pertinente lorsque l'œuvre peut être ramenée à un énoncé critique sur la société de consommation, que celui-ci soit manifeste ou latent. Dans ce dernier

I. Son premier roman, Le Méridien de Greenwich, est paru aux Éditions de Minuit en 1979.

2. J.-Cl. Lebrun, Jean Echenoz, Éditions du Rocher, coll. «Domaine français», I992, p. 2I.

3. J. Baudrillard, Le Système des objets [1968], Gallimard, coll. «Tel», I978.

4. G. Genette, «Structuralisme et critique littéraire», dans Figures I [1966], Seuil, coll. «Points Essais», I976, p. I55. 
cas cependant, le choix d'une telle lecture est encore sujet à caution s'il aplanit les stratégies énonciatives qui brouillent et minorent cet énoncé critique, n’hésitant pas à réduire au statut d'œuvre "assertive» le roman qui a peut-être avant tout «une valeur interrogative», lorsqu'il est, pour l'écrivain, la manière «à la fois [d']engager profondément son ouvre dans le monde, dans les questions du monde, mais aussi [de] suspendre cet engagement précisément là où les doctrines, les partis, les groupes et les cultures lui soufflent une réponses».

Or, dans les romans de Jean Echenoz, rien ne laisse penser, justement, que les objets puissent supporter de manière privilégiée le discours de la démystification, si l'on entend d'abord celui-ci comme le projet de dévoiler les mécanismes cachés conférant aux objets un sens et une valeur dans la logique de la consommation. Certes, ils sont parfois des traces, dans le roman, du caractère excessivement périssable des produits de la société urbaine contemporaine, vite démodés, dégradés, et mis au rebut. Les «chopes publicitaires dont s'écaill[e] parfois la décalcomanie" parsèment souvent les espaces domestiques ${ }^{6}$, et le mobilier urbain, inégalement réparti dans le métro parisien, est l'occasion pour le narrateur d'Au piano de s'interroger : "quatre poubelles direction Étoile alors que seulement deux direction Nation, pourquoi? Jetterait-on moins quand on revient des beaux quartiers ${ }^{7}$ ?» Dans les quartiers aisés de L'Équipée malaise, les éboueurs sont ironiquement les chevaliers de la modernité triomphante, chargés d'effectuer «le tour d'honneur des balayures, l'adieu à la valeur d'usage, à la valeur d'échange, avant la décharge puis l'incinération ${ }^{8} »$. Mais comme le dit lui-même Echenoz, ce qui est intéressant dans l'objet, "c'est la dimension émouvante de l'usure, de l'érosion de l'objet, son histoire, son incongruité, sa séduction un peu paradoxale». En ce sens, les objets quotidiens «sont à la fois sensuels et pouvant être de petits détonateurs de fiction", et l'on peut donc "avoir un rapport un peu amoureux aux choses ${ }^{9} »$. Les objets de ses romans se prêtent donc mal à une lecture axiologique catégorique et autoritaire. Ils caractérisent à eux seuls la polysémie de ces textes, dont Stéphane Chaudier dit très justement que le style $\mathrm{y}$ « rend un hommage ambigu au monde qu'il représente; il s'en sépare par la conscience aiguë de ses limites. Mais cette conscience est-elle plutôt

5. R. Barthes, «La littérature, aujourd'hui» [196I], Essais critiques, Seuil, coll. «Points Essais», I98I, p. 165.

6. J. Echenoz, L’Équipée malaise [1986], Minuit, coll. "Double», 2000, p. 22.

7. Id., Au piano, Minuit, 2003, p. 75.

8. Id., L'Équipée malaise, op. cit., p. 225.

9. Ces citations sont extraites de l'entretien que l'auteur nous a accordé le 2I février 2006, reproduit en annexe de notre thèse, Le Jeu des objets dans le roman français contemporain - Une expérience de la quotidienneté, D. Rabaté (dir.), université Michel-de-Montaigne - Bordeaux 3 , 2009, p. 455-469. 
acceptation ou dénonciation? Une telle ambivalence envers l'ordre des choses

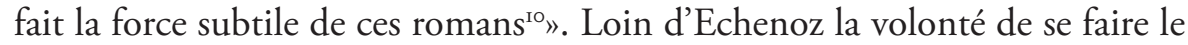
chantre de la société de consommation et pourtant, en pratique, ce sont les objets de celle-ci qui sont l'occasion d'un renouveau romanesque. L'écriture échenozienne s'approprie le réel par l'expérimentation de ses composantes les plus insignifiantes, elle est avant tout un art de l'exploration des interstices.

Plutôt que les repères rigides d'un système aliénant, les objets sont ainsi le lieu du roman où se dévoile l'existence d'un jeu dans ce système, si l'on entend le jeu au double sens de ce qui est ludique et de ce qui témoigne d'un petit décalage, où s'amorce ainsi la possibilité d'une «invention du quotidien $^{\mathrm{II}}$ ». Pour autant, l'attention portée aux objets n'est pas la métamorphose, l'enchantement ou la sacralisation des presque-rien du quotidien. Il ne s'agit pas de transformer l'anodin en extraordinaire, l'insignifiant en sur-signifiant mais, comme le disait plutôt Georges Perec, de «tenter de saisir, non ce que les discours officiels (institutionnels) appellent l'événement, l'important, mais ce qui est en-dessous». Entendre l'infra-ordinaire, le «bruit de fond qui constitue chaque instant de notre quotidiennetér ${ }^{12} »$. Les objets sont en effet la marque de la présence, dans le roman, d'une forme d'insignifiance qui n'est pas nécessairement le symptôme d'une vacuité de l'expérience quotidienne, mais d'une invitation à un pivotement du regard dont le goût du narrateur pour la notation s'avère le meilleur indice. L'insignifiant sur lequel ne cesse de se poser le narrateur échenozien, à la suite duquel s'engouffre le lecteur, s'apparente à ce que Barthes définissait comme le romanesque et estimait impossible avec le roman, "un mode de discours qui n'est pas structuré selon une histoire; un mode de notation, d'investissement, d'intérêt au réel quotidien, aux personnes, à tout ce qui se passe dans la vie ${ }^{13}$ ». Jean Echenoz, dont les romans sont pourtant essentiellement à la troisième personne du singulier, joue d'un système de contrepoint énonciatif, lequel permet précisément au roman d'accueillir une telle forme de romanesque. La voix du narrateur est si présente qu'on a pu parler à son égard d' «évidence du narrateur ${ }^{\mathrm{r}}$ », et

Io. S. Chaudier, «Michon, Echenoz : un parallèle», Jean Echenoz: "une tentative modeste de description du monde”, Chr. Jérusalem et J.-B. Vray (dir.), Saint-Étienne, Publications de l'université de Saint-Étienne, 2006, p. 67.

II. M. de Certeau, L'Invention du quotidien - I. Arts de faire [1980], Gallimard, coll. "Folio », I990 [nouvelle édition, établie et présentée par L. Giard].

I2. J.-M. Le Sidaner, «Entretien avec Georges Perec» [ $L^{\prime} A r c, n^{\circ}$ 76, 1976], Éditions Inculte, 2005 , p. 28.

I3. R. Barthes, "Vingt mots-clés pour Roland Barthes» [entretien avec J.-J. Brochier, I975], Le Grain de la voix, Seuil, coll. «Points Essais», I999, p. 239.

I4. G. Rubino, "Lévidence du narrateur", Jean Echenoz : "une tentative modeste de description du monde", op. cit., p. 22I-229. 
dire que "compte tenu de l'inconsistance moyenne des personnages, hommes sans qualité, c'est lui le véritable protagoniste ${ }^{15}$ ». Ce narrateur, présent au point qu'on a parfois l'impression de lire un roman à la première personne $^{\mathrm{I} 6}$, note dès qu'il le peut ce qui relève du "réel quotidien", de "tout ce qui se passe dans la vie». Il ne se pose jamais en détenteur d'un savoir total comme le narrateur balzacien, par exemple, mais en usager lui-même de la ville, dépositaire d'un savoir partiel mais attesté par l'expérience, qui prend souvent les apparences d'un mode d'emploi de la vie quotidienne urbaine, comme dans cet exemple extrait de L'Équipée malaise : si l'on descend à pied le Faubourg-Saint-Antoine, il est bien de faire

[...] une brève halte à la hauteur du 53, d'où le génie de la Bastille n'a plus l'air juché sur sa colonne que les immeubles dissimulent entièrement : il semble marcher sur leurs toits, danser sur leurs tuiles, sur leur zinc, exhibant dans sa fuite ses fesses rondes sous ses ailes déployées. Tout le monde sait cela, les gens s'arrêtent souvent devant le $53^{17}$.

Il note aussi volontiers, lorsque ses personnages passent devant sans plus s'en apercevoir, que les antennes paraboliques, sur les balcons, sont "orientées dans la même direction comme des champs verticaux de tournesols ${ }^{18}$ ", ou que la station de métro Bel-Air peut être vue, pour un regard curieux, comme "une île qui surplomberait en oasis la rue du Sahel dépeuplée ${ }^{19}$ ". Le narrateur s'aperçoit encore que des glaçons «bondi[ss]ent de leur étui de caoutchouc", avant de "grelotter ensuite dans le gin ${ }^{20}$ ". Les romans d'Echenoz, qui attirent sans cesse notre regard sur un détail ou un objet dont la signification paraît faible à l'échelle du roman, sont ainsi très visuels. Des effets de répétition, comme ceux que permet le procédé de l'anadiplose, provoquent souvent un ralentissement de l'action ou de la description. On peut comprendre ainsi un tel passage de L'Équipée malaise: "C'était encore un très mauvais mardi pour Paul, [...] assis sur l'extrême bord du plus manvais fautenil. Le plus manvais fautenilvomissait par en dessous des spires d'oxyde et de la paille verte, des lambeaux de jute corrompu ${ }^{21}$.» L'état piteux dans lequel se trouve le personnage que sa femme vient de quitter a déjà été mentionné à de

15. Ibid., p. 227.

16. Indépendamment des quelques cas où le narrateur intervient pour commenter le récit, à la première personne, notamment dans Je meen vais, Minuit, 2000, p. I89 : "Personnellement je commence à en avoir un peu assez, de Baumgartner. Sa vie quotidienne est trop fastidieuse."

17. J. Echenoz, L'Équipée malaise, op. cit., p. I27.

18. Id., Au piano, Minuit, 2003, p. 70.

I9. Ibid., p. 74.

20. J. Echenoz, L'Équipée malaise, op. cit., p. I8.

2I. Ibid., p. 38-39. 
nombreuses reprises. L'anadiplose du "plus mauvais fauteuil» semble ici ne faire qu'attirer l'œil du lecteur sur ce fauteuil, comme pour simplement laisser sentir l'évidence de sa présence. De même que le texte nous invite souvent à fixer brièvement notre attention sur un objet ou un mouvement, de même tend-il parfois à se focaliser sur une succession de gestes, laquelle fragmente l'action en une suite de moments paraissant indépendants les uns des autres, et décompose les automatismes. L'énumération prend alors une valeur paratactique, comme dans cette évocation du démarrage de sa voiture par Meyer dans Nous trois : "Contact, starter, moteur, ceinture, première, autoradio ${ }^{22}$." La logique d'inventaire, à laquelle se livre volontiers aussi un narrateur qui supplante sans vergogne son personnage absorbé à ce moment-là dans de tout autres pensées, et engagé dans d'autres actions, semble parfois relever de la distraction, tant elle est effectuée avec nonchalance, mais s'avère en dernier ressort une marque de cet intérêt pour "tout ce qui se passe dans la vie», par l'attention prêtée à l'apparemment insignifiant. L'inventaire des objets présents sur les plages arrière des voitures, dans Nous trois, au moment où Meyer se dirige vers sa propre voiture, en serait assez emblématique :

Les voitures garées dans l'impasse ont l'air vides mais on aperçoit, sur leur plage arrière, quelques journaux pliés, des cartes et guides routiers, parapluies et catalogues, boîte de kleenex et petits ventilateurs ou par exemple une peluche décorative décolorée, un chapeau vert, un gant vert, un listing d'ordinateur, l'édition de poche d'un roman d'Annabel Buffet, rarement plus d'une ou deux de ces choses en même temps ${ }^{23}$.

L'inventaire se présente d'abord comme le produit d'une observation directe, que le narrateur réalise pour le seul lecteur, puisque Meyer ne remarque nullement ces détails. Mais cet inventaire se pare subitement de nonchalance par l'usage curieux d'un "par exemple» qui renvoie l'ensemble de l'énumération à son caractère assez aléatoire. Feignant ensuite d'anticiper un éventuel malentendu sur ce sujet d'importance, le narrateur précise in fine que l'ensemble de ces objets ne se trouvent bien sûr pas tous dans la même voiture - l'adverbe «rarement» ayant d'abord une valeur litotique - comme s'il souhaitait conserver un semblant d'ordre dans le désordre d'une liste non exhaustive et glisser discrètement du cas particulier de cette observation à une conclusion d'ordre général. Il n'y a donc pas de lien autre que celui créé par le hasard entre tous les objets de ce pseudo-inventaire. Ces objets énumérés suggèrent, par leur accumulation plus que par leurs significations propres, combien on n'épuise jamais l'expérience quotidienne urbaine même si, au premier abord, les espaces considérés paraissent vides. Le refus d'une posture

22. J. Echenoz, Nous trois, Minuit, I992, p. I6.

23. Idem. 
totalisante et la légèreté adoptée par l'observateur conferent ainsi à chaque objet relevé tout aussi bien une existence propre qu'une relative insignifiance. Volontiers enclin à laisser croire qu'il lâche le fil de son récit, le narrateur fait alors entendre sa voix pour le simple plaisir de souligner l'intérêt qu'il porte à une multitude de détails à première vue secondaires et sans nécessité apparente. Dominique Viart, qui qualifie l'écriture d'Echenoz d' "éthique de la minutie $\mathrm{e}^{24}$, écrit d'ailleurs que «la pertinence romanesque n'a [...] que peu à voir avec l'économie diégétique. Elle s'accroche plutôt à tout élément susceptible d'être mis en relief par l'écriture ${ }^{25} »$. Ainsi, l'histoire que les romans d'Echenoz "prétendent raconter n'est finalement que prétexte à notations marginales, fragmentaires, saisies du réel quotidien ${ }^{26}$ ».

Est-ce à dire que les romans d'Echenoz, entachés d'une forme de gratuité ou de légèreté, sont dénués de toute portée sociale? Bien au contraire. Mais la place qu'ils accordent à la vie quotidienne et à ses objets les engage dans une voie oblique de résistance aux pesanteurs et aux carcans de la vie urbaine contemporaine plutôt que dans celle de la démystification frontale toujours suspecte de dogmatisme. Dans la perspective ouverte par Perec, prêter attention à la vie quotidienne, aux "choses communes ${ }^{27}$ ", c'est regarder le commun et voir l'en-commun. L'inscription privilégiée, dans le discours du narrateur échenozien, du thème des pratiques et des usages quotidiens, la prise en considération des objets ordinaires et les commentaires qui les élèvent à la dignité romanesque en établissant une connivence avec le lecteur, sont les traces, dans le roman, de la mise au jour d'une communauté d'expérience propre aux sujets contemporains. L'audace d'un narrateur qui n'hésite pas à se détacher de son personnage, à l'encontre de toutes les lois de l'économie narrative, en déclarant :

Comme il ne se passe pas grand-chose dans cette scène, on pourrait l'occuper en parlant de ce ticket. C'est qu'il y aurait pas mal de choses à dire sur ces tickets, sur leurs usages annexes - cure-dents, cure-ongles ou coupe-papier [...] - et leurs divers destins - pliés en deux ou en quatre dans le sens de la longueur et susceptibles alors d'être glissés sous une alliance, une chevalière, un bracelet-montre $[\ldots]^{28}$.

n'a pas qu'un effet de légèreté comique, ou d'expressivité de l'intériorité nerveuse du héros, Max Delmarc. Elle revient aussi à considérer que le regard

24. D. Viart, «Entretien avec Bruno Blanckeman», Prétexte, "États de la prose», printemps I999, p. IOI.

25. Id., "Le divertissement romanesque, Jean Echenoz et l'esthétique du dégagement", Jean Echenoz: "une tentative modeste de description du monde», op. cit., p. 249.

26. Ibid., p. 248.

27. G. Perec, «Approches de quoi?» [1973], L'Infra-ordinaire, Seuil, coll. «La Librairie du $\mathrm{XX}^{\mathrm{e}}$ siècle», I989, p. II.

28. J. Echenoz, Au piano, op. cit., p. 7 I. 
porté sur la variété des pratiques et des usages, quels que soient les signes distinctifs du personnage principal, a une dignité romanesque pour la seule raison que le lecteur y redécouvrira sa propre expérience à l'égard des objets. Selon Marcel Gauchet, "l'individu contemporain aurait en propre d'être le premier individu à vivre en ignorant qu'il vit en société, le premier individu à pouvoir se permettre, de par l'évolution même de la société, d'ignorer qu'il est en société29». Les romans d'Echenoz mettent certes en scène, à travers plusieurs de leurs personnages principaux, des individus solitaires et fuyants, peu enclins à considérer le lien social comme une donnée majeure de leur existence : il n'est qu’à penser au Meyer de Nous trois qui, «vient se garer non loin du gros fumigène, [mais] c'est moins par souci de son prochain que de lui-même, c'est surtout pour se changer les idées». Mais les romans d'Echenoz, quoique fils de leur époque, se découvrent plutôt discrètement, grâce à la communauté d'expérience pointée et instaurée par le contrepoint d'une voix narrative s'intéressant «au réel quotidien, aux personnes, à tout ce qui se passe dans la vie», comme des tentatives de résistance du sujet contemporain à l'atomisation des expériences, plutôt que de simples reflets d'un état de société auquel ils ne sauraient que se résigner.

L'abondance des objets et des notations insignifiantes dans ces romans pose nécessairement la question de la place, de l'émergence et de la persistance du sujet. Celui-ci paraît en effet sans cesse menacé, et le quotidien se fait tout autant le lieu de cette menace que de sa possible conjuration. Pourquoi la vie quotidienne contemporaine est-elle devenue propice à la mise au jour de telles tensions? Les travaux du sociologue Henri Lefebvre, pour une part concomitants de ceux de Barthes et de Perec, proposaient dans les années soixante une explication socio-historique : la vie quotidienne étant essentiellement une vie urbaine, le caractère coercitif de l'organisation sociale s'y fait d'autant plus ressentir, et limite les possibilités d'expression du sujet. Henri Lefebvre met particulièrement l'accent sur l'uniformisation des modes de vie en ville et écrit que, de ce fait, notre "vie quotidienne ne possède plus aucun style ${ }^{30}$ ». Le quotidien est cependant, pour lui, le lieu de toute aliénation mais aussi de toute libération possible. La ville est ainsi l'espace de l'expérimentation d'une quotidienneté renouvelée, où le sujet peut tenter de se frayer un chemin singulier. Elle est l'aire où se joue constamment la réversibilité de l'en-commun et de l'uniforme. Les romans du quotidien sont donc d'abord des romans urbains, de sorte que les textes où entrent en jeu tant d'objets

29. M. Gauchet, La Démocratie contre elle-même. Essai de psychologie contemporaine, I, Gallimard, coll. «Tel», 2002, p. 254.

30. H. Lefebvre, Critique de la vie quotidienne, t. II, Fondement d'une sociologie de la quotidienneté [1962], L'Arche, coll. "Le Sens de la marche», 1980, p. 321. 
sont de fait un espace littéraire où s'expérimentent les modalités de la vie en ville. Rendant sensible, grâce aux objets en particulier, l'expérience de la quotidienneté contemporaine, les romans d'Echenoz proposent aussi d'envisager des configurations possibles de la vie urbaine, de ses écueils et de ses enjeux.

L'omniprésence de l'insignifiance et de la ténuité, dont témoigne la saturation de ces romans par les objets, nous détourne de la recherche des mécanismes cachés qui régissent le grand dispositif social et que dévoilerait la fiction, et nous aiguille plutôt vers la mise au jour des modalités subtiles de cette entreprise de conciliation de la vie individuelle et de la vie collective, prolongeant pour cela les propositions avancées par Barthes au Collège de France lorsqu'il cherche Comment vivre ensemble ${ }^{31}$. Pour lui, c'est le concept d'idiorrythmie qui nourrit la problématique et apparait comme «le mot révélateur d'un fantasme latent de sociabilité32 ». Comme le note Claude Coste dans sa préface à l'édition du cours de Barthes, " [s]ans lien direct avec la vie conventuelle, l'idiorrythmie désigne [...], dans le cours de Barthes, toutes les entreprises qui concilient ou tentent de concilier [...] l'indépendance du sujet et la sociabilité du groupe ${ }^{33}$ ", elle "renvoie à toute communauté où le rythme personnel de chacun trouverait sa place ${ }^{34} »$. Claude Coste montre que la quête de Barthes est celle d'un lieu utopique "où espace géographique et espace social se confondent dans une même topique de la distance. Tout le cours est dans cette question : à quelle distance dois-je me tenir des autres pour construire avec eux une sociabilité sans aliénation, une solitude sans exil35?». Si le cours ne propose évidemment pas de réponse, il nous semble que les romans d'Echenoz sont eux aussi des variations sur cette interrogation.

En effet, au quotidien, cette problématique de la distance a trait à celle des objets, d'au moins deux manières. La première est celle qui permet à Barthes de reprendre à son compte la notion de proxémie ${ }^{36}$. Celle-ci relève d'une «typologie des espaces subjectifs en tant que le sujet les habite effectivement ${ }^{37}$ ». C'est la proximité spatiale de ces objets avec le sujet au quotidien qui les érige en objets familiers, producteurs d'affects, grâce auxquels le sujet

3I. R. Barthes, Comment vivre ensemble. Simulations romanesques de quelques espaces quotidiens - Cours et séminaires au collège de France (1976-1977), texte établi, annoté et présenté par Cl. Coste, Seuil/IMEC, coll. "Traces écrites», 2002.

32. Ibid., préface de Cl. Coste, p. 24.

33. Ibid., p. 25.

34. Ibid., p. 24.

35. Ibid., p. 28.

36. La proxémie est l'ensemble «des observations et théories concernant l'usage que l'homme fait de l'espace en tant que produit culturel spécifique». La définition, extraite du Dictionnaire des sciences sociales, est citée par Barthes lors de la "Séance du 20 avril I977", Comment vivre ensemble, op. cit., p. I55.

37. R. Barthes, Comment vivre ensemble, op. cit., p. 155 . 
peut s'isoler et faire retour sur lui-même. La seconde articule de manière plus vaste les objets et la vie en ville. Prenant acte de ce que «dans notre monde (celui industrialisé de la société dite de consommation) : ce qui coûte cher, le bien absolu, c'est la place», soulignant que «le bien luxueux, c'est d'avoir autour de soi de la place, c'est-à-dire "quelques-uns" mais peu : problème typique de l'idiorrythmie», Barthes imagine une nouvelle règle thélémite, calquée sur la règle monastique :

[...] règles de saint Benoît : l'abbé donne en propre à chaque moine des objets [...] selon le besoin vital; minimum nécessaire et significatif (car à cette époque, ce qui coûte, donc objet de don : les objets fabriqués). Eh bien, aujourd'hui, la règle thélémite ne donnerait plus des objets (trop facile, trop peu de valeur pour constituer un don consacrant), mais de la place ${ }^{38}$.

L'attention à l'omniprésence des objets suscite en retour le questionnement critique sur l'espace et les distances. Ceux-ci sont primordiaux dans la perspective ouverte par la tension idiorrythmique. La vie en ville et son organisation sociale tendent en effet à imposer leur cadence propre. Or, comme le rappelle Barthes, étymologiquement, le rythme renvoie au mouvement et non à la cadence. D'une part, «idiorrythme» constitue ainsi presque un pléonasme, car le rhuthmos est par définition individuel : interstices, fugitivité du code, de la manière dont le sujet s'insère dans le code social (ou naturel)». D'autre part, il «renvoie aux formes subtiles du genre de vie», il est «le contraire même d'une cadence cassante, implacable de régularité. C'est parce que le rythme a pris le sens répressif [...] qu'il a fallu lui adjoindre idios ${ }^{39}$ ». Si la vie urbaine est du côté de la cadence, on conçoit aisément que la quotidienneté urbaine soit une notion particulièrement problématique, puisqu'une vie véritablement quotidienne - à la fois commune et singulière - s'inscrit au contraire au creux du rythme propre au sujet. L'expérimentation d'une quotidienneté urbaine se fera donc à travers la quête d'un rhuthmos urbain, d'une exploration des interstices de la vie urbaine, du jeu fugitif avec ses codes.

Dès son premier roman, Jean Echenoz pointe d'ailleurs la difficulté qu'il peut y avoir à vivre au quotidien selon son rythme propre, en mettant en scène un personnage, Byron Caine, qui préfère échapper à toute vie quotidienne plutôt que d'en subir l'emprise potentiellement aliénante. Ce choix n'est bien sûr pas sans conséquences sur sa santé mentale, puisqu'en fuyant l'emprise de la vie quotidienne, il renonce dans le même temps au cadre sécurisant que celle-ci peut constituer, et à la marge de création et d'affirmation du sujet qu'elle pourrait offrir s'il s'en emparait :

38. Ibid., p. I79, pour ces dernières citations. 39. Ibid., p. 39, pour ces dernières citations. 
Byron Caine existait à vrai dire sans attache sensible, sans ancrage particulier. Ne s'attardant ni aux objets ni aux décors, il traversait l'espace avec une inattention sincère. Jamais il n'avait pu acquérir la notion de domiciliation, se mouler à l'impératif civique du lieu privé, intime, adhésif. [...] [I]l n'avait jamais cessé de neutraliser obstinément ses logements successifs, dans une lutte à mort contre la personnalisation [...]

Ainsi, à Paris, son bureau du boulevard Haussmann et son appartement de la rue Pétrarque, pôles rigoureux d'une quotidienneté binaire, lui étaient également familiers et étrangers, intimes autant qu'extimes, semblables en cela, par exemple, à une cabine d'ascenseur, à la salle d'attente d'une dentiste [...]

Mais c'était justement l'époque où l'inventeur n'inventait plus rien ${ }^{40}$.

Contrairement à l'extrême attention que le roman lui-même prête aux détails et aux objets, son personnage central se caractérise par l'«inattention sincère » qu'il adopte à leur égard. On pourrait d'abord croire que Byron Caine ne refuse que le repli dans l'espace domestique, auquel renverrait «la notion de domiciliation». Il s'agirait alors pour lui d'un refus de la survalorisation $\mathrm{du}$ «chez-soi». Byron Caine ressent d'ailleurs «inévitablement» une «sorte de saturation fade, [un] écœurement sucré ou plutôt saccharin" à l'occasion de "tout séjour un peu prolongé», "où que ce fût "4 ${ }^{4}$. L'inventeur subvertirait ainsi les normes et les périls d'une quotidienneté réduite à l'intimité. Mais, mettant sur le même plan l'espace de son domicile et tous les autres espaces urbains qu'il est amené à traverser ou à fréquenter, Caine s'oppose surtout à ce qu'il considère comme un «impératif civique du lieu privé». Cette curieuse formule laisse entrevoir combien le refus de l'ancrage dans le quotidien et dans la matérialité de son décor s'appuie d'abord sur le refus du vivre-ensemble. L'impératif "civique", avec sa connotation politique, renvoie bel et bien aux règles de vie dans la cité, lesquelles ne sont qu'une autre façon de désigner les nécessités de la vie urbaine, et les compromis qu'elle exige. Malgré la radicalité de sa stratégie d'évitement des pesanteurs de la vie quotidienne urbaine, ou en raison d'elle, plutôt, il en est paradoxalement venu à subir une "quotidienneté binaire", laquelle ne garde de la vie en ville que l'aspect répressif. Refusant la distinction de l'intime et de l'extime, du quotidien domestique et du quotidien urbain, il a, de fait, renoncé à l'affirmation de son rythme propre dans les mailles de la cadence urbaine. Sa résistance au quotidien se résout significativement dans la défaite de toute quotidienneté créatrice, puisqu' elle correspond à «l'époque où l'inventeur n'inventait plus». S'exilant ensuite avec sa compagne sur une île déserte, enrôlés dans la réalisation d'un mystérieux "projet Prestidge", Caine connaît de nouveau un cuisant échec : «Mais voilà, à peine arrivés sur l'île, la situation avait séché

40. J. Echenoz, Le Méridien de Greenwich, Minuit, 1979, p. 215-2I8.

4I. Ibid., p. 218-219. 
sur pied comme un plan inarrosé. Au double, triple jeu, succéda l'absence de jeu; à l'effervescence, la répétition; au rotor, le stator ${ }^{42}$." En renonçant à la vie en ville, au vivre-ensemble et à ses contraintes, les deux protagonistes ont aussi renoncé à l'intersubjectivité favorisée par la vie urbaine, et s'ennuient, s'assèchent, s'appauvrissent; leur vie se mécanise définitivement, le quotidien leur pèse irrémédiablement. Se tenant trop loin des autres, ils ne sont certes plus aliénés par eux, mais leur solitude n'est plus désormais qu'un véritable exil.

Si Le Méridien peut être lu comme la matrice de l'œuvre à venir, on ne sera pas étonné de voir ensuite le romancier déployer à nouveaux frais les modalités de cette quête de la bonne distance. Celle-ci se manifeste de manière privilégiée à travers les rituels de politesse qui scandent les situations quotidiennes évoquées par le romancier. De manière générale, c'est bien la trop faible distance, physique et psychique, ménagée entre les individus par la vie urbaine, qui rend indispensable l'obsession de la politesse. Parmi tant d'autres occurrences, on peut relever cette "politesse promiscue» organisant les échanges entre les passagers de l'ascenseur d'un centre commercial, laquelle "régit également les relations, sans condiment, des tranches de rosbif serrées dans un tupperware au frigo" dans Nous trois ${ }^{43}$, la stratégie d'évitement des "gênes promiscues» mise en œuvre, dans Je m’en vais, par Baumgartner. Dans le métro, ce dernier opte toujours pour les strapontins qui lui évitent de

[...] se trouver assis à côté de quelqu'un ou en face de quelqu'un, le plus souvent d'ailleurs les deux en même temps. Ce qui induirait encore des frottements et des gênes, des contacts, des difficultés de croisement ou de décroisement des jambes, des regards parasites et des conversations dont il n'a que faire ${ }^{44}$.

$\mathrm{Ou}$ encore ces scènes d'agressivité qu'offre le «spectacle urbain» dans ce même roman, lorsque deux «jeunes femmes [...] s'entre-égorgent pour une place de parking ${ }^{45}$ ", oubliant évidemment ici, justement, toute civilité.

La problématique idiorrythmique se traduit en fait de manière privilégiée, chez Jean Echenoz, par une attention relativement désenchantée aux situations urbaines où pourrait se jouer, à travers les interactions et les rituels de politesse, l'invention de modalités du vivre-ensemble qui préservent la singularité du sujet tout autant que le lien l'unissant à ses concitoyens. Mise en échec, au sein de la diégèse, par la cadence urbaine, l'idiorrythmie reste néanmoins l'horizon du roman grâce au contrepoint délicat qu'y instaure la voix narrative. Celle-ci règle en effet en permanence la distance qu'elle adopte

42. Ibid., p. 220.

43. J. Echenoz, Nous trois, op. cit., p. 67.

44. Id., Je m'en vais, op. cit., p. 92.

45. Ibid., p. 143. 
à l'égard de ses personnages tout aussi bien que de ses lecteurs. En laissant contaminer sa langue par celle de la parole quotidienne, le roman du quotidien pose aussi les limites de la familiarité qu'il s'y autorise. La création d'une atmosphère quotidienne passe en effet en partie par l'usage d'un lexique et d'une syntaxe souvent eux-mêmes pour le moins décontractés. Les objets quotidiens sont d'ailleurs particulièrement révélateurs de cet usage consensuel de la familiarité : référents communs de l'ère de la consommation de masse et de la société de l'information, leur possession, leur usage, et leur nomination, n'ont pas de caractère distinctif. Si ces objets sont communs, leur désignation familière traduit l'en-commun de leur pratique. Mentionner la "télé», le «frigo» ou les «godasses» constitue pour les écrivains du quotidien l'un des moyens les plus évidents pour évoquer, chez le lecteur, les pratiques d'une quotidienneté marquée par l'évidence et la décontraction. Pour autant, ces indices linguistiques de la communauté de l'expérience quotidienne ne sont pas exclusifs d'une forme de résistance à l'uniformisation des modes de vie et de pensée. Le quotidien reste, à travers la langue du roman, aussi bien le lieu du commun que du singulier. Le lexique et la syntaxe vont et viennent constamment entre cette proximité avec le lecteur qu'est la familiarité, et la distance que constitue une certaine manière, qui peut parfois sembler tendre au maniérisme. C'est que ces romans prennent acte du décor et du mode de vie urbains, pour tenter de faire émerger, au sein des normes et de l'uniforme, la part d'inventivité qui traduit la présence du sujet. Le lexique et la syntaxe se relâchent souvent en épousant les habitudes linguistiques contemporaines les plus communes, les moins particularisantes. Mais cette connivence instaurée à travers cet usage de la langue est bornée, contenue dans les limites de la réserve et des réserves qu'y oppose notamment la précision lexicale d'un narrateur que caractérise une "éthique de la minutie». Selon le philosophe Guillaume Le Blanc, et dans la perspective des travaux menés par Michel de Certeau, la vie ordinaire est «toujours triomphe de l'homme normal mais avec persistance de l'homme nomade ${ }^{46} »$. N'est-ce pas surtout cette vie ordinaire que donnent à percevoir les romans d'Echenoz, en prêtant attention au jeu des objets quotidiens, et en s'efforçant, à travers celui-ci, d'instaurer les conditions littéraires d'une communauté idiorrythmique avec les lecteurs?

Etre attentif à cette inflation de l'insignifiance, et refuser de systématiser le relevé de ces realia, c'est donc accepter le retournement du regard et de la lecture que programment ces textes. Si les objets, dans ces romans, participent d'une opération de démystification, c'est de celle qui vise à débusquer le banal,

46. G. Le Blanc, Les Maladies de l'homme normal, Bègles, Éditions du passant, 2004, p. 188 . 
que le psychanalyste Mahmoud Sami-Ali définit comme le littéral, «la formule qui l'exprime $(\mathrm{A}=\mathrm{A})$ est l'évidence même de ce qui reste identique à soi ${ }^{47}$ ». Il est "ce familier qui, à force de familiarité, n'a plus rien à voir avec l'étrange ${ }^{48}$ ", et a "partie lié avec l'épuisement du contenu émotionnel et cognitif de l'objet, moyennant une répétition qui ne manque pas d'engendrer un équivoque sentiment de monotonie ${ }^{49} »$. Démystifier le banal, c'est mettre au jour les forces coercitives qui s'exercent au quotidien à l'encontre du sujet citadin, ces normes de pensée et de comportement productrices d'une cadence urbaine qui risquent à tout instant de pétrifier le sujet. À moins que, comme le lui proposent les romans d'Echenoz, il trouve sa place et la bonne distance à l'égard de ce monde d'objets, pour y introduire du jeu, propre à favoriser grâce à la projection de l'imaginaire, la résistance au banal, cette réduplication du même et ce pseudo-naturel qui ne sont peut-être rien d'autre que cette «agression de la bêtise ambiante, intramondaine», qu'évoquait Barthes.

47. M. Sami-Ali, Le Banal, Gallimard, coll. «Connaissance de l'inconscient », 1980, p. 24. 48. Ibid., p. I9.

49. Ibid., p. 23-24. 